\title{
ON THE ESTIMATION OF POLARIMETRIC PARAMETERS FOR OIL SLICK FEATURE DETECTION FROM HYBRID POL AND DERIVED PSEUDO QUAD POL SAR DATA
}

\author{
Hari Priya $\mathrm{S}^{1, *}$, Jayasri PV ${ }^{1}$, SitaKumari EVS ${ }^{1}$, Prasad AVV ${ }^{1}$ \\ ${ }^{1}$ National Remote Sensing Centre, ISRO, Hyderabad, India - (haripriya_s, jayasri_pv, sitakumari_evs, prasad_avv)@nrsc.gov.in
}

\author{
Commission V, SS: Emerging Trends in Remote Sensing
}

KEY WORDS: SAR, RISAT-1, CTLR, pseudo-quad pol, correlation coefficient, sigma naught

\begin{abstract}
Oil spills in oceans have a significant long term effect on the marine ecosystem and are of prime concern for maritime economy. In order to locate and estimate the oil spread area and for quantitative damage assessment, it is required to continually monitor the affected area on the sea and its surroundings and space based remote sensing makes this technically viable. Synthetic Aperture Radar SAR with its high sensitivity to target dielectric constant, look angle and polarization -dependent target backscatter has become a potential tool for oil-spill observation and maritime monitoring. From conventional single-channel SAR (single-pol, HH or VV) to multi-channel SAR - (Dual/Quad-polarization) and more recently compact polarimetric (Hybrid/Slant Linear) SAR systems have been widely used for oil-spill detection in the seas. Various polarimetric features have been proposed to classify oil spills using full, dual and compact polarimetric SAR. RISAT-1 is a C-band SAR with Circular Transmit and Linear Receive (CTLR) hybrid polarimetric imaging capability. This study is aimed at the polarimetric processing of RISAT-1 hybrid pol single look complex (SLC) data for derivation of the decisive polarimetric parameters which can be used to identify oil spills in oceans and their discrimination from look-alike signatures. In order to understand ocean -oil spill signatures from full-quad pol SAR, pseudo-quad pol covariance matrix is constructed from RISAT-1 hybrid pol using polarimetric scattering models. Then polarimetric processing is carried out over pseudo-quad pol data for oil slick detection. In-house developed software is used for carrying out the above oilspill study.
\end{abstract}

\section{INTRODUCTION}

India has a long coastline and a thriving maritime economy. Oil-slick incidents near Mumbai, the recent Ennore oil-spill and the likes, call for immediate investigation and damage control measures. A large amount of oil is yearly discharged into the marine environment due to vessel operations and land-based sources of petroleum pollutants because of which coastal environments may suffer from long term damage to ocean flora and fauna. Thus maritime monitoring on a regular basis using space based remote sensing has become one of the primary requirements. The theoretical rationale of SAR oil spill detection is that the presence of oil slicks on the sea surface dampens short-gravity and capillary waves, so the Bragg scattering from the sea surface is largely weakened.*

Synthetic Aperture Radar SAR is an active microwave imaging sensor having all-time, all-weather imaging capability coupled with wide area coverage, polarization diversity and lesser revisit time. Fully polarimetric SAR involves coherent transmission and reception of linear Horizontal $\mathrm{H}$ and Vertical V signals and hence present a complete picture about the target area but at the cost of architectural complexity and reduced swath coverage. In recent times, compact polarimetric CP SAR has evolved as an alternate solution as it offers reduced complexity, cost, mass and data transmission rate while retaining the relevant polarimetric information of the target. Circular transmit/linear receive (CTLR) SAR is a compact hybrid pol system which transmits right or left circular polarization and receives linear $\mathrm{H}$ and V. Hybrid pol SAR has the advantages of having a greater amount of polarization information than the standard dual-pol linear systems while overing twice the swath width of quad-pol SAR systems, thus

\footnotetext{
* Corresponding author
}

making it a potential tool for carrying out ocean-based studies including oil-spill detection and maritime monitoring. The main effect of oil on the ocean surface is the dampening of small-scale ocean surface waves, the Bragg waves, which reduce electromagnetic backscatter from areas where oil is present, generating relatively darker zones in the SAR image, and therefore allowing detection (M. Migliaccio 2015). The presence of oil on the ocean surface reduces surface tension and friction between the wind and the water surface. Conventional oil spill detection procedures use intensity, morphological texture and auxiliary information .However, while dealing with oil spill detection, look-alike signatures caused by natural biogenic films produced by marine organisms, grease ice, low wind areas, rain cells, shear zones, internal waves and ship wakes have to be delineated as these look-alikes can limit the conventional oil spill remediation services (Brekke and Solberg, 2005).

Polarimetric SAR systems have the capability to separate the contributions of different scattering elements available in a single SAR resolution cell. Different polarimetric features can be derived or transformed from the target scattering matrix. Thus polarimetric SAR can be used to derive the required oil spill detection parameters and provide an unambiguous mechanism to discard look-alike features.

RISAT-1 SAR, operating in the C-band, has the compact hybrid polarimetric imaging capability. Transmission is done in Right Circular mode and coherent linear reception is possible giving rise to RH and RV output channel datasets. This study has been carried out as follows:

1.Polarimetric processing of RISAT-1 circular Fine Resolution Stripmap data (cFRS1) hybrid pol data and estimation of the relevant oil spill parameters like degree of polarization, 
ellipticity angle, complex correlation coefficient, conformity coefficient and sigma naught backscatter coefficients for oilslick covered,clean sea-surface and look-alike signatures in the data.

2. Analysis and implementation of compact polarimetric scattering models and reconstruction technique for conversion of RISAT-1 to pseudo-quad polarimetric data and derivation of quad polarimetric oil slick features using in-house developed software.

3. Quantitative comparison of the derived parameters and their capability to distinguish between oil spill and look-alike signatures.

\section{COMPACT HYBRID POLARIMETRIC SAR}

The primary motivation for compact polarimetric CP-SAR is for advocating quantitative backscatter classifications and target identification at the same finesse as those from a fully polarimetric system .CP SAR is configured with relatively simple architecture, low cost, and wide swath coverage as compared to Quad pol SAR. Hybrid polarimetric SAR is a class of CP-SAR where either right or left circular polarization is transmitted, while both the linear orthogonal polarizations are received with their relative phase information intact. Such a configuration is referred to as Circular Transmit Linear Receive configuration or CTLR SAR.

The fundamental data product from CTLR hybrid SAR is the complex target vector of the backscattered field. However in most analyses of hybrid-pol SAR, the real focus is on the Stokes parameters $\left(S_{1}, S_{2}, S_{3}\right.$ and $\left.S_{4}\right)$ of the scattered wave (Stokes, 1852, Raney, 2007; Cloude et al., 2012) which are derived from the complex scattering matrix of the received field. This is because of the fact that in the case of partially polarized waves it is easier to use Stokes parameters as these parameters characterise partially polarized waves not by their phases and amplitude but by their observable power terms.

For the CTLR case, where the transmission is right circular and reception is linear, the Stokes' parameters can be expressed as (R.Raney 2007)

$$
\begin{aligned}
& S_{1}=\left\langle\left|E_{R H}\right|^{2}+\left|E_{R V}\right|^{2}\right\rangle \\
& S_{2}=\left\langle\left|E_{R H}\right|^{2}-\left|E_{R V}\right|^{2}\right\rangle \\
& S_{3}=2 \operatorname{Re}\left\langle E_{R H} E_{R V}^{*}\right\rangle \\
& S_{4}=-2 \operatorname{Im}\left\langle E_{R H} E_{R V}^{*}\right\rangle
\end{aligned}
$$

Where $E$ is the complex voltage, $*$ denotes complex conjugate and $\langle\ldots\rangle$ indicates ensemble averaging in a suitable window size, by assuming the wave as stationary.

\subsection{Hybrid Polarimetric Parameters for Oil Slick Detection}

Hybrid CTLR SAR has been extensively used as a potential tool in representing an interesting operational alternative for detecting oil slicks and discriminating them from weakdamping surfactants . This is possible owing to its coherent polarimetric information content. For oil covered areas in the oceans and seas, the polarimetric scattering mechanisms are different . Oil layer suppresses Bragg scattering when compared to the surrounding water. This scattering effect is largely observed in the derived values of Stokes parameters .
Effective oil spill detection is commonly based on the total power of the four polarimetric channels, physically corresponding to the first Stokes parameter, $S 1$ (Liu et al., 2011). By comparison, the $S 3 / S 4$ ratio can be more generally used for target detection on the ocean surface $(\mathrm{Li} \mathrm{H}$ et al., 2012). On the ocean surface, the co-polarization correlation is much larger than correlations between the co and crosspolarization channels (Nghiem et al., 1992). The primary oil slick feature detection parameters can be derived from the four Stokes parameters. The degree of polarization $(\mathrm{m})$ is a key parameter ( Shirvany et al.) and has been used to detect oil spills, ships, and buoys using $\mathrm{CP}$ data.

The degree of polarisation ' $\mathrm{m}$ ' can be derived as

$$
m=\frac{\sqrt{\mathbf{S 2}^{2}+\mathbf{S 3}^{2}+\mathbf{S 4}^{2}}}{\mathbf{S 1}}
$$

Relative Phase ( $\delta$ ): Relative phase is the phase between RH and $\mathrm{RV}$ and can be calculated using Stokes Parameters as

$$
\delta=\tan ^{-1}\left(\frac{S 4}{S 3}\right)
$$

The Ellipticity parameter has been employed as an indicator of the presence of dominant Bragg scattering and is defined as:

$$
\operatorname{Sin}(2 \chi)=-\left(\frac{\mathrm{S} 4}{\mathrm{~m} * \mathrm{~S} 1}\right)
$$

where ' $\chi$ ' is the ellipticity angle varying in the range $\left(-45^{0}\right.$ to $+45^{\circ}$ ). This indicates the presence of odd versus even -bounce backscattering. As Bragg scattering due to presence of oil slick gives rise to single-bounce reflection, positive $\chi$ values are retuned by oil-slick region and negative $\chi$ values are observed for the unaffected ocean region. Thus, the ellipticity factor can be used to differentiate Bragg and non-Bragg regions in the area of interest.

Another polarimetric factor which can be directly derived from the hybrid pol SAR measurements is the conformity coefficient $\mu \mathrm{HP}$ (Zhang et al. 2011)

$$
\mu \mathrm{HP}=\frac{2\left(\zeta\left(\mathrm{E}_{\mathrm{hq}} \mathrm{E}_{\mathrm{vq}} *\right)\right)}{\left(<\left|\mathrm{E}_{\mathrm{hq}}\right|^{2}>+<\left|\mathrm{E}_{\mathrm{vq}}\right|^{2}\right\rangle}
$$

This parameter has the inherent ability to provide a binary output space in which sea surface gives rise to positive values and oil slicks give rise to negative values.

The complex correlation co-efficient between the two orthogonal received components can be defined as (Born and Wolf 1980; Nunziata,Migliaccio and Li 2015):

$$
|\xi| e^{j Q}=\frac{S 3+j S 4}{\sqrt{S 1+S 2} \sqrt{S 1-S 2}}
$$

where $\mathrm{Q}$ is the phase difference between the wave components along the $\mathrm{h}$ and $\mathrm{v}$ axes. A fully polarised wave is characterised by $|\xi|=1$ and a totally unpolarised wave gives $|\xi|=0$. For the clean sea surface the wave scattered off it, gives $|\xi|=1$ while the oil covered region gives $|\xi|$ to be nearly 0 .

\section{FULL QUAD POLARIMETRIC SAR}

Fully polarimetric or quad-pol SAR data comprises transmission of both linear $\mathrm{H}$ and $\mathrm{V}$ polarizations and coherent reception of co-pol and cross-pol information, thereby giving 
rise to four output channels. In this case, the elements of the scattering matrix are complex, and are obtained from the magnitudes and phases measured by the four channels of a polarimetric radar. The scattering matrix for the quad-pol configuration, on a linear horizontal $(\mathrm{H})$ and vertical $(\mathrm{V})$ basis, is given by

$$
[S]=\left[\begin{array}{l}
S_{H H} S_{H V} \\
S_{V H} S_{V V}
\end{array}\right]
$$

where the first subscript denotes the RX pol mode and the second subscript represents the TX pol mode of the scattering matrix.

It has been proved that for a given frequency and viewing geometry, the scattering matrix depends only on the scattering properties of the observed scene (Cloude 2009).

One of the primary methods to deal with polarimetric scattering from a distributed and depolarising scene is to use second order products of the scattering matrix(Van Zyl,Elachi 1987).According to the Stokes formalism, the scattered field is can be related to the incident field by the $4 \times 4$ real and symmetric Mueller matrix(Guissard 1994). The Mueller matrix representation is the most general representation of polarimetric scattering and can be constructed from the target scattering vector.

The scattering matrix can also be represented by the target vector $\boldsymbol{k}$ as :

$$
\vec{k}=\left[\begin{array}{lll}
S_{H H} & \sqrt{2} S_{H V} & S_{V V}
\end{array}\right]^{T}
$$

The related $3 \times 3$ covariance matrix $-\mathrm{C}$ matrix is generated from the outer product of the target vector with its conjugate transpose. Assuming backscattering alignment (BSA) , the expression for the covariance matrix is given as :

$$
C=\left\langle\begin{array}{ccc}
\left|S_{H H}\right|^{2} & \sqrt{2} S_{H H} S_{H H}^{*} & S_{H H} S_{V V}^{*} \\
\sqrt{2} S_{H V} S_{H H}^{*} & 2\left|S_{H V}\right|^{2} & \sqrt{2} S_{H V} S^{*}{ }_{V V} \\
S_{V V} S_{H H}^{*} & \sqrt{2} S_{V V} S_{H V}^{*} & \left|S_{V V}\right|^{2}
\end{array}\right\rangle
$$

where $*$ denotes the conjugate and $\langle>$ indicates ensemble averaging.

\subsection{Quad Polarimetric Parameters for Oil Slick Detection}

Many extensive studies have been done to formulate improved methodologies to detect and classify oil spills, based on different scattering mechanisms for oil on the ocean surface. The classification comparisons among dual-pol, reconstructed pseudo quad-pol from $\mathrm{CP}$, and quad-pol SAR data have been studied (Souyris et al., 2005; Ainsworth et al., 2009; Nord et al., 2009). Moreover, damping is always stronger for VV polarization, than for $\mathrm{HH}$ polarization (Franceschetti et al., 2002). Therefore, on a sea surface covered by oil, the intensity difference for the co-polarization mode is dramatically reduced.
The co-polarised correlation coefficient and the conformity co-efficient play a major role as oil-spill indicators in full pol SAR (Miglachio 2015 ) and are given as:

Co-pol correlation

$$
|\rho|=\left|\frac{S H H S V V *}{\left.\sqrt{\left\langle|S H H|^{2}\right.}\right\rangle \sqrt{\left\langle|S V V|^{2}\right\rangle}}\right|
$$

Conformity Coefficient $\mu=2 *\left(\operatorname{re}\left(\mathrm{S}_{\mathrm{HH}} \mathrm{S}_{\mathrm{VV}} *\right)-\left\langle|\mathrm{SHV}|^{2}\right) / \mathrm{Span}\right.$

where're' indicates real part and Span denotes the total power.

\section{RECONSTRUCTION OF PSEUDO QUAD POL FROM HYBRID POL DATA}

One of the common methods to compare hybrid pol and quad pol SAR data is to implement quad-pol construction which concentrates mostly on retrieving quad-pol SAR matrices from the second-order statistics of CP SAR measurement vector like the covariance matrix. To reconstruct the quad-pol data from $\mathrm{CP}$ SAR data by using an iteration-based algorithm, the magnitude of linear coherence and cross polarization ratio were linked with a parameter $\mathrm{N}=4$ (Souyris et al 2005). This was later modified using Nord's algorithm by replacing $\mathrm{N}$ with $\left(\left|\mathrm{S}_{\mathrm{HH}}-\mathrm{SVv}\right| 2 /\left|\mathrm{S}_{\mathrm{HV}}\right|^{2}\right)$ and updated it during the calculation process iteratively (N Nord 2009). However, because scatter behaviours of sea surface and vegetation-covered ground area differ significantly, neither Souyris' algorithm nor Nord's modified version could work well in their application to sea surface monitoring. Later an empirical model to estimate $\mathrm{N}$, at variance of incidence angles, by fitting the observed data with negative exponent function was devised (MJ Collins et al 2013).

The quad-pol reconstruction algorithm is physically based on the hypothesis of the reciprocity and reflection symmetry of geophysical SAR data (Souyris et al b 2005) i.e.,

$$
\mathrm{S}_{\mathrm{HV}}=\mathrm{S}_{\mathrm{VH}}(\text { Reciprocity) }
$$

Reflection symmetry is a property of media that are symmetric with respect to the incidence plane (Souyris 2005 ), which is consistent with many terrestrial imaging scenarios as well as imaging of the ocean in the upwind or downwind directions. Reflection symmetry implies complete de-correlation between the co-polarized and cross-polarized backscattering coefficients i.e

$$
\mathrm{S}_{\mathrm{HH}} \mathrm{S}_{\mathrm{HV}} *=\mathrm{S}_{\mathrm{VV}} \mathrm{S}_{\mathrm{HV}} *=0
$$

The pseudo-quad pol covariance matrix can be constructed from the received hybrid pol $\mathrm{RH}$ and $\mathrm{RV}$ (9) polarimetric scattering and recontruction models (JC Souyuris 2005, N Nord 2009,MJ Collins 2013). The idea is the conceptual interpolation between two extreme deterministic scattering scenarios: completely polarized backscatter and completely unpolarized backscatter (JC Souyris et al 2005). This interpolation relates the ratio of cross-pol-to-copol energy to the co-pol phase angle. In the Souris model, the proportionality is a constant, i.e. $N=4$. In the Nord model, the proportionality is, itself, dependent on the co-pol backscatter and cross-pol backscatter and must be iteratively solved.

The reconstruction of the quad-pol covariance matrix from hybrid pol data is a two step process. In order to estimate the quad-pol $3 \times 3$ covariance matrix, we must estimate nine unknowns. However, the hybrid pol mode provides us four elements of the covariance matrix. In order to derive the other unknowns, some assumptions are made to constrain the solution 
space and reduce the number of unknowns and/or increase the number of equations.

Step 1: Initialization:

$\left\langle\left|S_{H V}\right|^{2}\right\rangle=0$

$\rho h \mathrm{vvv}=\left(\frac{-\mathrm{jC} 12}{C 11 * C 22}\right)$

$\left\langle\left|\mathrm{S}_{\mathrm{HV}}\right|^{2}\right\rangle=\left(\frac{C 11 * C 22}{2}\right)\left(\frac{1-\rho h h v v}{\frac{N}{2}+(1-\rho h h v v)}\right)$

Step 2: Reconstruction of QP data:

$$
\begin{aligned}
& \left\langle\left|\mathrm{S}_{\mathrm{HH}}\right|^{2}\right\rangle=\mathrm{C}_{11}-\left\langle\left.\mathrm{SHV}\right|^{2}\right\rangle \\
& \left\langle\left|\mathrm{S}_{\mathrm{HV}}\right|^{2}\right\rangle=\mathrm{C}_{22}-\left\langle\left|\mathrm{S}_{\mathrm{HV}}\right|^{2}\right\rangle \\
& \left\langle\mathrm{S}_{\mathrm{HH}} * \mathrm{SVV}_{\mathrm{V}}\right\rangle=\left\langle\left|\mathrm{S}_{\mathrm{HV}}\right|^{2}\right\rangle-\mathrm{jC}_{12}
\end{aligned}
$$

where $\mathrm{C}_{11}, \mathrm{C}_{12}, \mathrm{C}_{21}$ and $\mathrm{C}_{22}$ are the hybrid pol covariance matrix elements derived from the Stokes parameters and phhvv is the co-pol correlation co-efficient of pseudo quad pol data.

\section{DATASETS}

The C-band RISAT-1 satellite launched in 2012 can be operated in Fine Resolution Stripmap -FRS-1 mode with Right Circular Transmit and coherent linear $\mathrm{H}$ and $\mathrm{V}$ reception giving RH and RV output data channels. The cFRS-1 Level-1 data is collected as Single Look Complex (SLC). The output channels are RH and RV data with amplitude and phase.

Oil slick dataset corresponding to the Norway Oil-slick along with two look-alike datasets were considered for the study.

\begin{tabular}{|c|c|c|c|}
\hline Mode & Orbit & $\begin{array}{c}\text { Date of } \\
\text { Imaging }\end{array}$ & $\begin{array}{c}\text { Incidence } \\
\text { Angle }\end{array}$ \\
\hline $\begin{array}{c}\text { cFRS-1 } \\
\text { (Oil-Slick) }\end{array}$ & $11810 / 11805$ & $17 / 06 / 2014$ & $49.4 \mathrm{deg}$ \\
\hline $\begin{array}{c}\text { cFRS-1(Look- } \\
\text { alike) }\end{array}$ & $11843 / 11847$ & $20 / 06 / 2014$ & $29.78 \mathrm{deg}$ \\
\hline $\begin{array}{c}\text { cFRS-1(Look- } \\
\text { alike) }\end{array}$ & $6203 / 6230$ & $11 / 06 / 2013$ & $38.95^{0}$ \\
\hline
\end{tabular}

\subsection{Methodology}

1. The RISAT- 1 cFRS-1 Single Look Complex is read and the real and imaginary $i$ and $q$ channels of the RH and RV datasets are segregated.

2. The Stokes parameters are derived from the RH and RV data. 3. In order to decrease the speckle effect, polarimetric refined Lee-Sigma filter with 5X5 sliding window is applied on the dataset.

4. As a first -cut approach for differentiating oil and water signatures, the sigma naught backscatter images are generated for $\quad$ RV and RH using in-house developed SigmaSAR-v3.1 utility.

5. The $2 \mathrm{X} 2$ polarimetric covariance matrix $\mathrm{C}$ is derived from the Stokes parameters.

6. The Stokes Child parameters $\mathrm{m}, \chi$, Conformity Co-efficient and Correlation Co-efficient are derived over small AOI regions over oil-slick and look-alike / clean sea
7. Pseudo-quad pol covariance matrix components $S_{H H}$ and $S_{V V}$ from $\mathrm{S}_{\mathrm{HV}}$ and complex co-pol correlation component $\left\langle\mathrm{SHHSvV}_{\mathrm{H}} *\right.$ are derived from hybrid pol covariance matrix elements.

8. Oil-slick parameters and look-alike parameters are compared with theoretical values.

\section{ANALYSIS AND RESULTS}

\subsection{Sigma Naught derivation}

As a preliminary step, the sigma naught backscatter values for the Single look complex RH and RV channels have been recorded over the oil slick and oil-free sea surface using inhouse developed SigmaSAR-v3.1 utility. The results observed are as below:

\begin{tabular}{|c|c|c|}
\hline $\begin{array}{l}\text { Sigma Naught } \\
\text { backscatter }\end{array}$ & $\begin{array}{c}\text { Oil-Slick } \\
\text { covered sea }\end{array}$ & $\begin{array}{c}\text { Slick-free sea } \\
\text { surface/Look-alike }\end{array}$ \\
\hline RV Channel & -25.9 & -13.0 \\
\hline & -18.0 & -19.3 \\
\hline & -30.2 & -21.7 \\
\hline & -20.0 & -17.9 \\
\hline RH Channel & -13.7 & -24.4 \\
\hline & -18.5 & -16.3 \\
\hline & -19.7 & -25.5 \\
\hline & -21.9 & -16.8 \\
\hline
\end{tabular}

Table 1: Sigma Naught Backscatter values for RH and RV (AOI based)

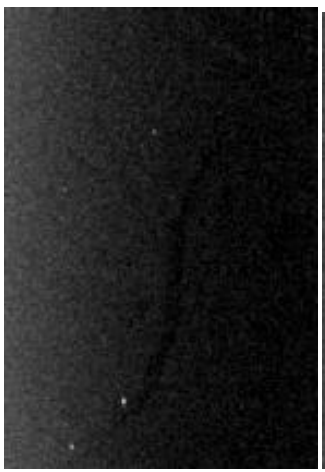

Sigma Naught-RH

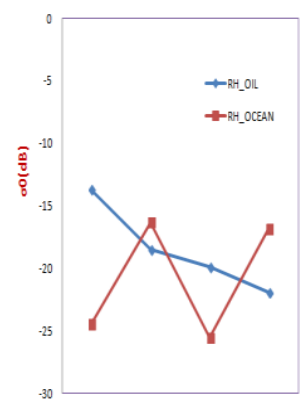

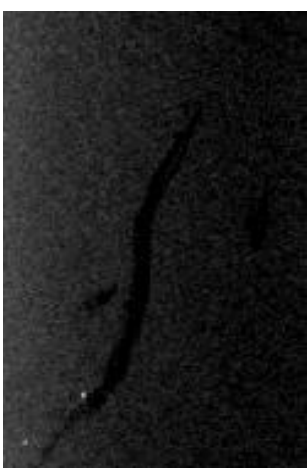

Sigma Naught-RV

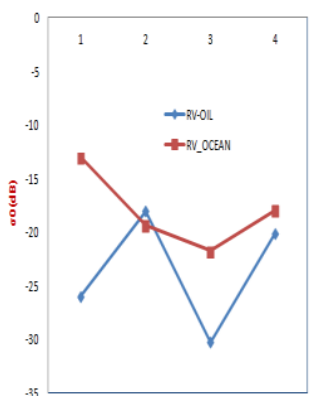

Figure 1: Sigma Naught Date of Imaging: 17-June 2014 Orbit: 11810/11805 Mode: cFRS-1 Incidence Angle: 49.40

It is observed that the RV channel gives better discrimination of oil slick signature when compared to RH channel. For one 
AOI , both RH and RV backscatter values differ only by a few $\mathrm{dB}$.

\subsection{Hybrid pol oil-slick parameters}

\begin{tabular}{|c|c|c|}
\hline $\begin{array}{c}\text { Hybrid } \\
\text { Polarimetric } \\
\text { Parameter }\end{array}$ & $\begin{array}{c}\text { Oil-Slick } \\
\text { covered sea }\end{array}$ & $\begin{array}{c}\text { Slick-free sea } \\
\text { surface/Look-alike }\end{array}$ \\
\hline \multirow[t]{3}{*}{$\begin{array}{c}\text { Degree of } \\
\text { Polarization-m }\end{array}$} & 0.454 & 0.792 \\
\hline & 0.499 & 0.640 \\
\hline & 0.477 & 0.705 \\
\hline \multirow[t]{3}{*}{$\begin{array}{l}\text { Circular Pol } \\
\text { Ratio(CPR) }\end{array}$} & 1.258 & 0.290 \\
\hline & 1.134 & 0.586 \\
\hline & 1.16 & 0.413 \\
\hline \multirow[t]{3}{*}{ 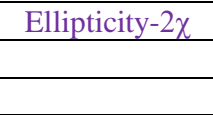 } & 0.006 & -0.719 \\
\hline & 0.012 & -0.305 \\
\hline & 0.019 & -0.188 \\
\hline \multirow[t]{3}{*}{$\begin{array}{l}\text { Complex } \\
\text { Correlation } \\
\text { Coefficient } \\
\end{array}$} & 0.376 & 0.737 \\
\hline & 0.358 & 0.622 \\
\hline & 0.322 & 0.449 \\
\hline \multirow[t]{3}{*}{$\begin{array}{c}\text { Conformity Co- } \\
\text { efficient }\end{array}$} & -0.041 & 0.577 \\
\hline & -0.03 & 0.531 \\
\hline & -0.05 & 0.408 \\
\hline
\end{tabular}

Table 2: Hybrid Polarimetric Parameters derived from RISAT-1 cFRS-1 for oil-slick and look-alike signatures(AOI-based)

For hybrid pol data, the degree of polarisation shows lower values for oil when compared to look-alike/clean sea because of Bragg scattering. The circular pol ratio is well above 1.0 for oil slick and between 0 and 1 for clean sea.The ellipticity factor is negative for clean sea and is positive for oil slick . Oilslick regions are showing lower copol correlation when compared to look alike /clean sea. The conformity coefficient is negative for oil-slick and positive for look-alike/ocean. These values are plotted as shown below and are in good agreement with previous experimental results.
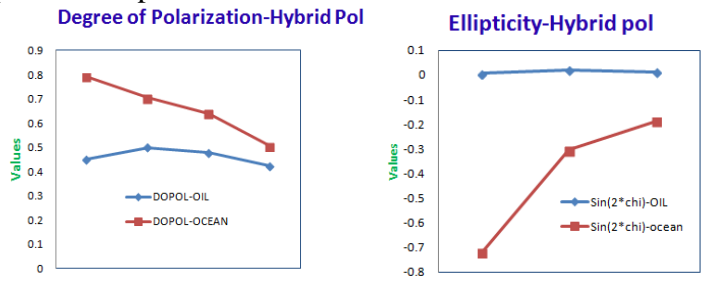

Circular Polarization Ratio-Hybrid Pol
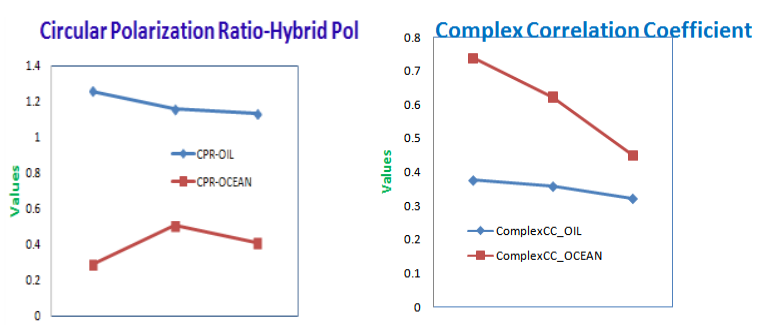

Hybrid Pol Conformity Coefficient

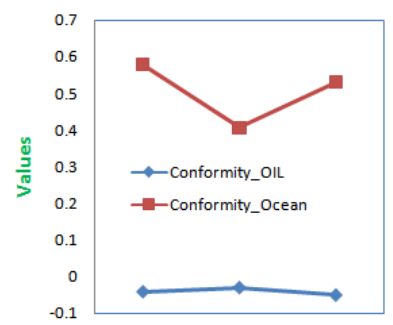

Figure 2: Plots showing the various hybrid pol oil-slick features considered in this study

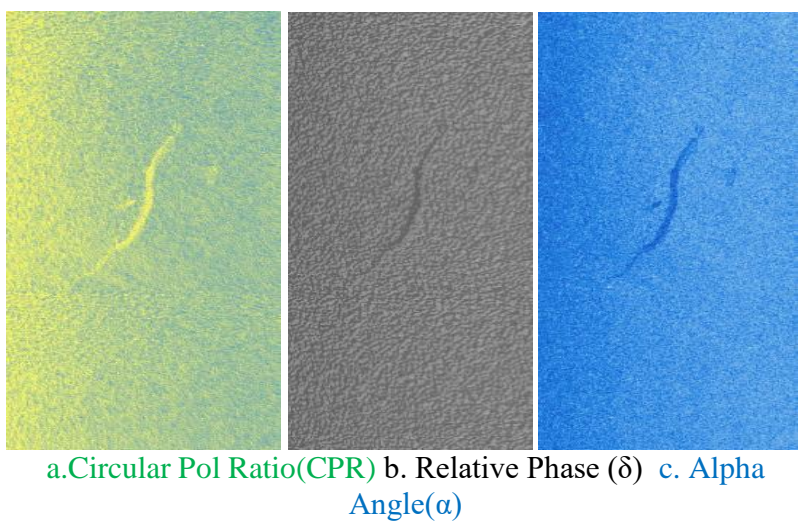

Figure 3: RISAT-1 CTLR hybrid Pol data with oilslick Date of Imaging: 17-June 2014 Orbit: 11810/11805 Mode: cFRS-1 Incidence Angle: $49.4 \mathrm{deg}$

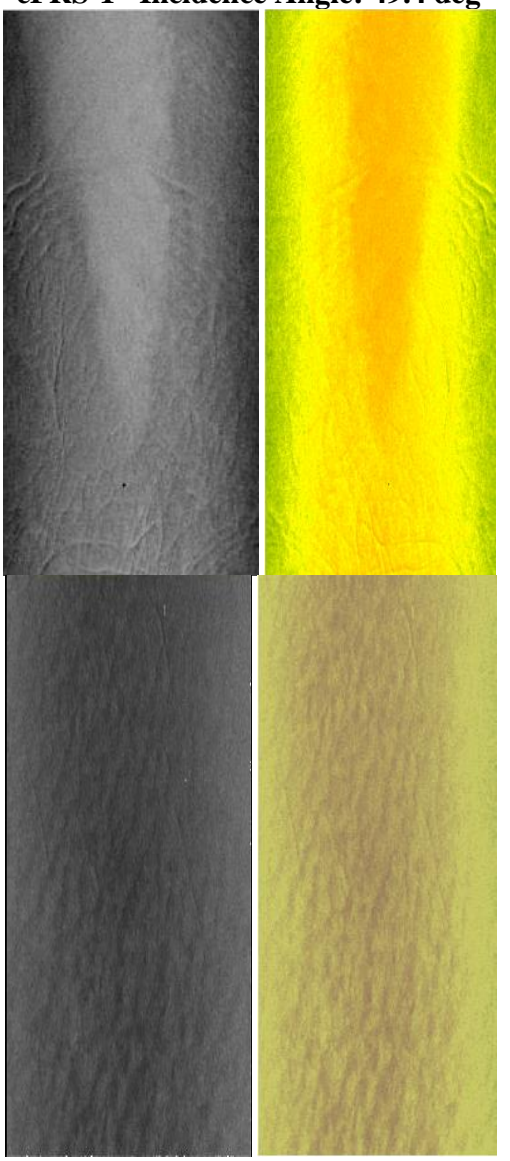

Figure 4: Look-alike signatures ( $T$ to $B$ ) $1 \mathrm{a}, 1 \mathrm{~b}$ Conformity Coefficient 2a, 2b- Correlation Coefficient 
1a,1b:Orbit 11843/11847 Incidence Angle: 29.7 deg; 2a,2b: Orbit:6203/6230 Incidence Angle:38.9 deg

\subsection{Pseudo-quad pol oil slick parameters}

\begin{tabular}{|c|c|c|}
\hline $\begin{array}{c}\text { Pseudo-Quad } \\
\text { Polarimetric } \\
\text { Parameter }\end{array}$ & $\begin{array}{c}\text { Oil-Slick } \\
\text { covered sea }\end{array}$ & $\begin{array}{c}\text { Slick-free sea } \\
\text { surface/Look-alike }\end{array}$ \\
\hline$\langle|\mathbf{S V V}|\rangle^{*}\langle|\mathbf{S V V}|>$ & 105957 & 200780 \\
\hline & 60691 & 249748 \\
\hline & 143788 & 271631 \\
\hline & 104442 & 287620 \\
\hline $\begin{array}{c}\text { Complex } \\
\text { Correlation } \\
\text { Coefficient }\end{array}$ & 0.191 & 0.641 \\
\hline & 0.093 & 0.726 \\
\hline & 0.231 & 0.479 \\
\hline & & 0.227 \\
\hline $\begin{array}{c}\text { Conformity } \\
\text { Coefficient }\end{array}$ & -0.142 & 0.261 \\
\hline & -0.069 & 0.564 \\
\hline & -0.037 & 0.301 \\
\hline
\end{tabular}

Table 3: Pseudo-Quad Polarimetric Parameters derived from RISAT-1 cFRS-1 for oil-slick and look-alikes

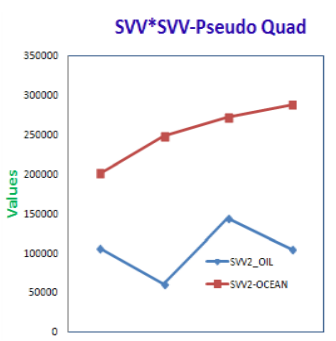

Copol Complex Correlation-Pseudo Quad

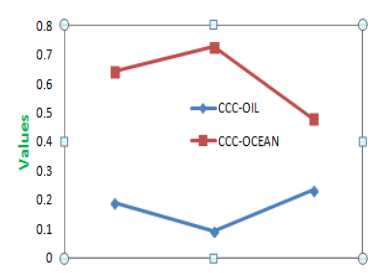

Conformity Co-efficient-Pseudo Quad

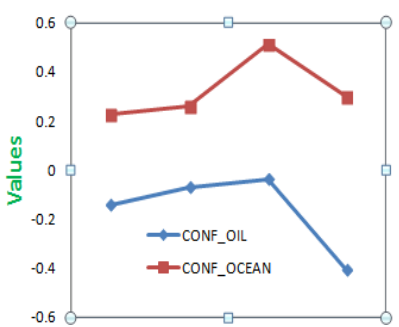

Figure 4: Pseudo-Quad polarimetric parameters for oil slick and ocean/look-alike signatures

The pseudo-quad pol covariance matrix is constructed and the relevant oil-spill parameters like $\left\langle\mathrm{S}_{\mathrm{Vv}}{ }^{2}\right\rangle$, complex correlation coefficient and conformity are derived and tabulated.The values of $\left\langle\mathrm{Svv}^{2}\right\rangle$ are lower for oil-slick and higher for lookalikes because of damping caused by oil on the V-pol component.The copol correlation is lesser in the case of oil and more in the case of look-alike/clean sea.The conformity is negative for oil and positive for the ocean surface.All these observations are in accordance with theoretical values and experimental results.

\section{CONCLUSIONS}

Oil slick detection in oceans and differentiating them from look -alike signatures pose a major challenge for environmental monitoring and polarimetric SAR act as a potential tool in this area. Although oil spill detection and classification can be achieved with quad-pol SAR, hybrid- pol SAR provides a wonderful alternate with its wide swath coverage and relatively simple architecture. RISAT-1 hybridpol SAR mode transmits in circular polarization, and receives in orthogonal linear polarizations, with information about the relative phase of the components. The polarimetric processing of RISAT-1 Circular FRS-1 complex SAR data has been carried out using in-house developed software, to derive the relevant oil-slick parameters like degree of polarization,Circular Pol Ratio, complex correlation and conformity coefficients and ellipticity angle on a AOI basis. The values for oil slick and look-alikes are clearly separable and can be easily used to separate oil covered region from look-alikes. In order to compare the above results with quad pol, reconstruction of pseudo-quad pol parameters from RISAT-1 hybrid pol covariance matrix has been carried out using polarimetric scattering and reconstruction models using in-house developed software. Then oil slick parameters are compared and are found to be in conformance with the theoretical values.

7.1 Future Scope: This work can be extended to draw comparisons between different pseudo-quad reconstruction techniques and select the one with better results. The oil slick and look-alike detection mechanism from polarimetric SAR can be made operational for continual maritime monitoring

\section{ACKNOWLEDGMENT}

The authors are immensely grateful to Shri. Shantanu Choudhary , Director ,NRSC Deputy Director , for the scientific motivation. We are immensely thankful to Shri.Vinod Bothale, Deputy Director DPPA\&WAA for his encouragement for research proposals. We are extremely grateful to Shri. AVV. Prasad, Group Director, MRS\&GDPG for guiding us with his technical expertise and encouraging us to work in various SAR related domains. Our sincere thanks are due to Smt. EVS. SitaKumari, Head, MRSDPD for her valuable technical inputs for improvising and for giving us a free hand in carrying out this research work. We extend our thanks to all our MRSDPD team members for their timely guidance and technical support.

\section{REFERENCES}

M. Migliaccio, F. Nunziata \& A. Buono (2015) SAR polarimetry for sea oil slick observation, International Journal of Remote Sensing, 36:12, 3243-3273, DOI: 10.1080/01431161.2015.1057301

J.-C. Souyris, P. Imbo, R. Fjørtoft, S. Mingot, and J.-S. Lee, "Compact polarimetry based on symmetry properties of geophysical media:The $/ 4$ mode," IEEE Trans. Geosci. Remote Sens., vol. 43, no. 3, pp. 634-646, Mar. 2005.

M. Nord, T. Ainsworth, J.-S. Lee, and N. Stacy, "Comparison of compact polarimetric synthetic aperture radar modes," IEEE Trans. Geosci. Remote Sens., vol. 47, no. 1, pp. 174-188, Jan. 2009. 
R. Raney, "Hybrid-polarity SAR architecture," IEEE Trans. Geosci. Remote Sens., vol. 45, no. 11, pp. 3397-3404, Nov. 2007. [4] R. Raney, "Comments on hybrid-polarity SAR architecture,” in Proc. IEEE IGARSS, 2007, pp. 2229-2231.]

On the Reconstruction of Quad-Pol SAR Data From Compact Polarimetry Data For Ocean Target Detection

Michael J. Collins, , Michael Denbina and Ghada Atteia, IEEE Transactions On Geoscience And Remote Sensing, Vol. 51, No. 1, January 2013

S. V. Nghiem, S. H. Yueh, R. Kwok, and F. K. Li, "Symmetry properties in polarimetric remote sensing," Radio Sci., vol. 27, no. 5, pp. 693-711, Sep./Oct. 1992

S. Yueh, S. Nghiem, and R. Kwok, "Comparison of a polarimetric scattering and emission model with ocean backscatter and brightness measurements," in Proc. IEEE IGARSS, 1994, vol. 1, pp. 258-260.

Skrunes, S.; Brekke, C.; Eltoft, T. An experimental study on oil spill characterization by multi-pol polarizationSAR. In Proceedings of the $9^{\text {th }}$ European Conferenceon Synthetic Aperture Radar(2012EUSAR),Nuremberg, Germany, 23-26 April 2012; pp. 139-142.

Shirvany, R.; Chabert, M.; Tourneret, J.-Y. Ship and oil-spill detection using the degree of polarization in linear and hybrid/compact dual-pol SAR. IEEE J. Sel. Top. Appl. Earth Obs. Remote Sens. 2012, 5, 885-892.

Salberg, A.-B.; Rudjord, O.; Solberg, A.H.S. Model based oil spill detection using polarimetric SAR. In Proceedings of the IEEE International Geoscience and Remote Sensing Symposium (IGARSS), Munich, Germany, 22-27 July 2012; pp. 5884-5887. 